\title{
NORMAL SEMIGROUPS OF ONE-TO-ONE TRANSFORMATIONS
}

\author{
by INESSA LEVI
}

(Received 24th January 1989)

\begin{abstract}
Let $X$ be an infinite set and $S$ be a transformation semigroup on $X$ invariant under conjugations by permutations of $X$. Such $S$ is termed $\mathscr{G}_{X}$-normal. In the paper, we describe elements of a $\mathscr{G}_{X}$-normal semigroup $S$ of one-to-one transformations.
\end{abstract}

1980 Mathematics subject classification (1985 Revision): 20M20.

\section{Introduction}

If a transformation semigroup $S$ is defined by means of certain properties there is a problem of determining the elements of $S$ explicitly. In this paper, the above problem is considered for $\mathscr{G}_{X}$-normal semigroups $S$ of total one-to-one transformations of a set $X$. A transformation semigroup $S$ on $X$ is termed $\mathscr{G}_{X}$-normal if $h S h^{-1} \subseteq S$ for every $h \in \mathscr{G}_{X}$, the symmetric group on $X$. For example, the semigroup $\mathscr{T}_{X}$ of all total transformations of $X$, the semigroup $\mathscr{I}_{X}$ of all one-to-one transformations of $X, W_{X}=\mathscr{T}_{X} \cap \mathscr{F}_{X}$ are $\mathscr{G}_{X}$-normal semigroups. Amongst some interesting properties of $\mathscr{G}_{X}$-normal semigroups is the fact that all their automorphisms are inner [2].

For a transformation $f$ of $X$, let $R(f) \quad(=f(X))$ denote the range of $f$, def $f=|X-R(f)|$ be the defect of $f$ and $|\{x \in X: f(x) \neq x\}|$ be the shift of $f$. We show that a $\mathscr{G}_{X}$-normal semigroup $S$ of one-to-one transformations contains almost all transformations that shift not more than $\alpha$ points of $X$ (for some infinite cardinal $\alpha$ ) and have defects of certain cardinality (Theorems 2.7 and 2.17). The result of Theorem 2.7 was announced (without a proof) in [3].

If $X$ is finite and $S$ is a $\mathscr{G}_{X}$-normal semigroup of one-to-one transformations of $X$, then $S \subseteq \mathscr{G}_{X}$. A $\mathscr{G}_{X}$-normal subsemigroup of $\mathscr{G}_{X}$ is a subgroup of $\mathscr{G}_{X}$ and is referred to as a normal subgroup of $\mathscr{G}_{X}$. A description of such groups can be found in [4]. Thus, we restrict our attention to infinite $X$ and permutation-free $S$. Moreover, it is sufficient to consider semigroups consisting of transformations with either only finite or only infinite defects, since any $S$ is either of one of the above types or a disjoining union of two semigroups of the above types.

\section{Cyclic-chain decomposition}

Our aim in this section is to decompose an arbitrary one-to-one transformation $f$ of 
$X$ into elementary and easily-studied components. When $f$ is a permutation, the elementary components are finite and infinite cycles and the decomposition is a (formal) cyclic decomposition [4, 1.3.4]. If $f$ is an arbitrary one-to-one transformation, then further components, chains (Definition 1.2), appear in a decomposition of $f$ (Proposition 1.4).

For a transformation $f$ of $X$, let $S(f)=\{x \in X: f(x) \neq x\}$. The shift of $f$ is the cardinality of $S(f)$. Transformations $f, g$ are said to be disjoint if $S(f) \cap S(g)=\Phi$, where $\Phi$ is the empty set. The following definition is a generalization of a formal product of permutations $[4$, p. 10].

Definition 1.1. Let $A$ be a set of pairwise disjoint one-to-one transformations of $X$. The formal product of the members of $A$ is a transformation of $X$ denoted by $\Pi\{f: f \in A\}$ and defined by the following:

$$
(\Pi\{f: f \in A\})(x)=\left\{\begin{array}{cl}
f(x) & \text { if } x \in S(f) \text { and } f \in A \\
x & \text { if } x \in X-\cup\{S(f): f \in A\}
\end{array}\right.
$$

where $x \in X$.

That a formal product of a set of pairwise disjoint permutations is a permutation is shown in $\left[4,1.3 .3\right.$.]. Similar arguments can be used to prove that if $A \subseteq W_{X}$, then $\Pi\{f: f \in A\} \in W_{\mathbf{X}}$.

Definition 1.2. If $x_{i} \in X, i=1,2, \ldots$ and $x_{i} \neq x_{j}$ for $i \neq j$ then $\left(x_{1} x_{2} x_{3} \ldots\right)$ denotes a transformation $f \in W_{X}$ such that $f\left(x_{i}\right)=x_{i+1}$ for $i=1,2, \ldots$ and $f(y)=y$ for all other $y \in X$. Such a transformation is called an $x_{1}$-chain, or just a chain.

The following is self-evident.

Lemma 1.3. Let $f$ be an $x$-chain, then $X-R(f)=\{x\}$ and $\operatorname{def} f=1$.

Proposition 1.4. Let $f$ be a non-identity transformation in $W_{X}$. Then $f$ is a formal product of pairwise disjoint cycles and chains, $f=\Pi\{g: g \in A\}$, with no $g \in A$ being $a 1$ cycle. The number of chains in $A$ is equal to $\operatorname{def} f$. If $f=\Pi\left\{g: g \in A^{\prime}\right\}$ is another such product then $A=A^{\prime}$.

Proof. Let $x \in X$ and $U(x)[L(x)]$ be the set of all distinct elements $f^{n}(x)$, where $n=0,1,2, \ldots[n=0,-1,-2, \ldots] . \quad$ Thus $U(x)=\left\{x, f(x), f^{2}(x), \ldots\right\}, \quad L(x)=\left\{x, f^{-1}(x)\right.$, $\left.f^{-2}(x), \ldots\right\}$.

With each $x \in S(f)$, we associate a transformation $h_{x}$ of $X$. The definition of $h_{x}$ depends on the sizes of $U(x)$ and $L(x)$. If $U(x)$ and $L(x)$ are both infinite, then $f^{n}(x) \neq x$ for every non-zero integer $n$ and we let $h_{x}=\left(\ldots f^{-1}(x), x, f(x), \ldots\right)$; clearly $h_{x}$ is an infinite cycle. If $U(x)$ and $L(x)$ are both finite, let $h_{x}=\left(x, f(x), \ldots, f^{n-1}(x)\right)$ where $n>1$ is 
a minimal integer such that $f^{n}(x)=x$; then $h_{x}$ is a finite cycle. If $U(x)$ is infinite and $L(x)$ is finite, let $h_{x}=\left(f^{-n}(x), f^{-n+1}(x), \ldots, x, \ldots\right)$ where $n \geqq 0$ is a minimal integer such that $f^{-n}(x) \notin R(f)$; then $h_{x}$ is a $f^{-n}(x)$-chain. Finally, we remark that the assumption that $U(x)$ is finite while $L(x)$ is infinite implies that for some integer $n \geqq 1, f^{n}(x)$ is not in the domain of $f$, a contradiction to the fact that $f$ is a total transformation.

Let $A=\left\{h_{x}: x \in S(f)\right\}$. It is easy to see that if two transformations in $A$ are not disjoint, then they are equal. Thus $\Pi\left\{h_{x}: h_{x} \in A\right\}$ is a formal product of a set $A$ and $\Pi\left\{h_{x}: h_{x} \in A\right\}=f$.

Finally, Lemma 1.3 implies that there is a one-to-one correspondence between $y$ chains in $A$ and points $y$ in $X-R(f)$. This observation asserts the fact that the number of chains in $A$ is equal to $\operatorname{def} f$.

Since our aim is to describe all the elements of an arbitrary $\mathscr{G}_{X}$-normal $S \subseteq W_{X}$ and for every $f \in S$ its conjugate by any permutation of $X$ is again in $S$, we are naturally interested in the question when $f, g \in W_{X}$ are conjugates of each other, that is when $f=h h^{-1}$ for some $h \in \mathscr{G}_{X}$. Recall that two permutations of $X$ are conjugate if and only if they have the same number of cycles of each length in their cyclic decomposition. For arbitrary mappings in $W_{X}$, we require also the number of chains in their cyclic-chain decomposition to be the same, that is they have to have the same defects (Proposition 1.4).

For $f \in W_{X}, n=1,2,3, \ldots ; \infty$ let $\phi_{n}(f)$ denote the number of cycles of length $n$ in the cyclic-chain decomposition of $f$. Lèt $C h_{X} \subseteq W_{X}$ be the set of all formal products of disjoint chains. Then Proposition 1.4 assures that every $f \in W_{X}$ can be written as a disjoint product of $f_{1} \in \mathscr{G}_{X}$ and $f_{2} \in C h_{X}$.

Proposition 1.5. Let $f, g \in W_{X}$. Then $f, g$ are conjugate if and only if $\operatorname{def} f=\operatorname{def} g$ and $\phi_{n}(f)=\phi_{n}(g)$ for every $n=1,2,3, \ldots ; \infty$.

Proof. In view of $[4,1.3 .6,1.3 .11]$ we assume that $f$ and $g$ are not permutations and write $f=f_{1} f_{2}, g=g_{1} g_{2}$, where $f_{1}, g_{1} \in \mathscr{G}_{X}, f_{2}, g_{2} \in C h_{X}$ and $f_{1}$ and $f_{2}, g_{1}$ and $g_{2}$ are disjoint. We assume also that $S\left(f_{1}\right)=S\left(g_{1}\right)$ and $S\left(f_{2}\right)=S\left(g_{2}\right)$ (else choose a permutation $p$ of $X$ that maps $S\left(f_{1}\right)$ onto $S\left(g_{1}\right)$ and $S\left(f_{2}\right)$ onto $S\left(g_{2}\right)$ and replace $f$ with $\left.p f p^{-1}\right)$. By $[4,1.3 .6,1.3 .11]$ we can choose a permutation $q$ of $X$ such that $f_{1}=q g_{1} q^{-1}$ and $q$ fixes every element of $X-S\left(f_{1}\right)$. It suffices now to show that if def $f=\operatorname{def} g$ (or, equivalently, def $f_{2}=\operatorname{def} g_{2}$ ) then there exists a permutation $k$ of $X$ that fixes every element of $X-S\left(f_{2}\right)$ and $f_{2}=k g_{2} k^{-1}$. Then $q$ and $k, g_{1}$ and $k$ and $q^{-1}$ and $g_{2}$ are pairwise disjoint and so

$$
\begin{aligned}
f=f_{1} f_{2}=q g_{1} q^{-1} k g_{2} k^{-1} & =q g_{1} k q^{-1} g_{2} k^{-1} \\
& =q k g_{1} g_{2} k^{-1} q^{-1} \\
& =(q k) g(q k)^{-1}
\end{aligned}
$$


as required. To construct $k$ as above let $f_{2}=\Pi\{t: t \in A\}, g_{2}=\Pi\{s: s \in B\}, A$ and $B$ be sets of disjoint chains. Then $|A|=\operatorname{def} f_{2}=\operatorname{def} g_{2}=|B|$ and there is a bijection $\lambda: A \rightarrow B$. For each $x$-chain $t \in A$ define a bijection $k_{t}: S(t) \rightarrow S(\lambda(t))$ such that if $\lambda(t)$ is a $y$-chain then $k_{t}\left(t^{m}(x)\right)=(\lambda(t))^{m}(y), m=0,1,2, \ldots$ For an $x \in X$ let

$$
l(x)= \begin{cases}k_{t}(x) & \text { if } x \in S(t) \text { and } t \in A \\ x & \text { if } x \in X-S\left(f_{2}\right)\end{cases}
$$

Then $k=l^{-1}$ is the required permutation.

The following lemma follows from elementary properties of one-to-one transformations and an observation that an element of $W_{X}-G_{X}$ has an infinite shift.

Lemma 1.6. If $f, g \in W_{X}-\mathscr{G}_{X}$, then

(1) def $f g=\operatorname{def} f+\operatorname{def} g$;

(2) shift $f g \leqq$ shift $f+$ shift $g$;

(3) if shift $f \neq$ shift $g$, then shift $f g=\max \{$ shift $f$, shift $g$ \}.

\section{Normally generated semigroups}

If $F$ is any set of transformations of $X$ then the semigroup generated by the set all conjugates of the elements of $F$ is the smallest $\mathscr{G}_{X}$-normal semigroup containing $F$. We denote this semigroup by $\left\langle F: \mathscr{G}_{X}\right\rangle$ and call it the semigroup normally generated by $F$. Thus

$$
\left\langle F: \mathscr{G}_{X}\right\rangle=\left\langle\left\{h f h^{-1}: f \in F, h \in \mathscr{G}_{X}\right\}\right\rangle .
$$

For brevity in what follows we write $\left\langle\{f\}: \mathscr{G}_{X}\right\rangle$ as $\left\langle f: \mathscr{G}_{X}\right\rangle$. Every $\mathscr{G}_{X}$-normal $S$ is normally generated by its own elements and if $f \in S$ then $\left\langle f: \mathscr{G}_{X}\right\rangle \subseteq S$. Thus semigroups of the type $\left\langle f: \mathscr{G}_{X}\right\rangle, f \in S$, are the building blocks for $S$. Hence our goal now is to describe $\left\langle f: \mathscr{G}_{X}\right\rangle$ for $f \in W_{X}$. This is done in Propositions 2.6 and 2.13.

We start with the following lemmas.

Lemma 2.1. Let $f$ be a chain. Then there exists a chain $q$ with $S(f)=S(q)$ such that $q f=g_{1} g_{2}$ with $g_{1} \in \mathscr{G}_{X}, g_{2} \in C h_{X}, g_{1}$ and $g_{2}$ are disjoint, shift $g_{1}=\aleph_{0}, g_{1}$ fixes $|X|$ points of $\mathrm{X}-\mathrm{S}\left(\mathrm{g}_{2}\right)$ and is a (formal) product of 2-cycles.

Proof. Let $f=\left(a_{1} a_{2} a_{3} a_{4} \ldots\right), \quad q=\left(a_{4} a_{1} a_{2} a_{3} a_{8} a_{7} a_{5} a_{6} a_{12} a_{9} a_{10} a_{11} a_{16} a_{15} a_{14} a_{13} \ldots\right)$. Then $g_{1}=\left(a_{1} a_{3}\right)\left(a_{9} a_{11}\right)\left(a_{17} a_{19}\right) \ldots, g_{2}=\left(a_{2} a_{8} a_{10} a_{16} a_{18} a_{24} \ldots\right)\left(a_{4} a_{12} a_{20} a_{28} \ldots\right)$ and $g_{1}$ fixes $\left\{a_{7}, a_{13}, a_{14}, a_{15}, a_{21}, a_{22}, a_{23}, \ldots\right\} \cup(X-S(f))$. 
Corollary 2.2. For any $f \in W_{X}$ there exists $h \in \mathscr{G}_{X}$ such that if $h f h^{-1} f=g_{1} g_{2}$ with $g_{1} \in \mathscr{G}_{X}, g_{2} \in C h_{X}, g_{1}$ and $g_{2}$ are disjoint then shift $g_{1}=\aleph_{0}$ def $f$ and $\left|X-S\left(g_{2}\right)\right|=|X|$.

Proof. Choose a conjugate $h f h^{-1}$ of $f$ such that for every cycle in $f, h f h^{-1}$ contains the inverse of the cycle and for every chain $f^{\prime}$ in $f, h f h^{-1}$ contains a chain $q^{\prime}$ such that $S\left(f^{\prime}\right)=S\left(q^{\prime}\right)$ and $q^{\prime} f^{\prime}$ is a disjoint product of $g_{1}^{\prime} \in \mathscr{G}_{X}$ with shift $g_{1}^{\prime}=\kappa_{0}$ and $g_{2}^{\prime} \in C h_{X}$ so that $\left|X-S\left(q^{\prime} f^{\prime}\right)\right|=|X|$ (Lemma 2.1).

Lemma 2.3. Let $f \in C h_{x}$. Then $\forall n \in \mathbb{N} f^{n} \in C h_{X}$ with $S\left(f^{n}\right)=S(f)$.

Proof. Clearly $S(f) \supseteq S\left(f^{n}\right)$ and if $x \in S(f)$ with $f^{n}(x)=x$ then $f$ contains a finite cycle, a contradiction that shows the reverse inclusion.

Observe now that if $g$ is an $x_{1}$-chain, $g=\left(x_{1} x_{2} x_{3} \ldots\right)$ then $g^{n}$ is a product of $n$ disjoint chains,

$$
g^{n}=\left(x_{1} x_{n+1} x_{2 n+1} \ldots\right)\left(x_{2} x_{n+2} x_{2 n+2} \ldots\right) \ldots\left(x_{n} x_{2 n} x_{3 n} \ldots\right)
$$

Let $f$ be a formal product of disjoint chains, $f=\Pi\{g: g \in A\}$. Then $\forall g \in A, S\left(g^{n}\right)=S(g)$ so that $f^{n}=\Pi\left\{g^{n}: g \in A\right\}=\Pi\{q: q \in B\}$, where the elements of $B$ are the members of the cyclic-chain decompositions of all $g^{n}, g \in A$.

We adopt the following notation from [3]. Let $\alpha$ and $\beta$ be two cardinals with $\beta<\alpha \leqq|X|^{+}$, where $|X|^{+}$is the cardinal successor of $|X|$. Let

$$
S(X, \alpha, \beta)=\left\{f \in W_{X}: \text { shift } f<\alpha, \operatorname{def} f=\beta\right\} .
$$

If $\alpha>\aleph_{0}$ and $\beta \geqq \aleph_{0}$ then $S(X, \alpha, \beta)$ forms $\mathscr{G}_{X}$-normal semigroup. If $0<\beta<\aleph_{0}$ then $S$ $(X, \alpha, \beta)$ is not a semigroup. If $\alpha \leqq \aleph_{0}$ and $\beta \neq 0$ then $S(X, \alpha, \beta)$ is empty. If $\beta=0$ then $S(X, \alpha, \beta)$ is a normal subgroup of $\mathscr{G}_{X}$.

Now let $f \in W_{X}, f=f_{1} f_{2}$ with $f_{1} \in \mathscr{G}_{X}, f_{2} \in C h_{X}$ and $f_{1}, f_{2}$ be disjoint. Let $Z=$ $X-S\left(f_{2}\right)$ and

$$
\mathscr{H}(f)=\left\{\left.h\right|_{Z} \in \mathscr{G}_{Z}: h \in \mathscr{G}_{X},\left.h\right|_{S\left(f_{2}\right)}=\left.i\right|_{S\left(f_{2}\right)} \text { and } h f_{2}^{n} \in\left\langle f: \mathscr{G}_{X}\right\rangle \text { for some } n \in \mathbb{N}\right\}
$$

where $i$ is the identity transformation on $X$.

Lemma 2.4. If $Z \neq \Phi$ then $\mathscr{H}(f)$ is a normal subgroup of $\mathscr{G}_{Z}$.

Proof. Let $\left.h\right|_{Z},\left.p\right|_{Z} \in \mathscr{H}(f)$, then $h f_{2}^{n}, p f_{2}^{m} \in\left\langle f: \mathscr{G}_{X}\right\rangle$, for some $m, n \in \mathbb{N}$ and $h f_{2}^{n} p f_{2}^{m}=$ $h p f_{2}^{n+m}$ since $S\left(f_{2}^{n}\right)=S\left(f_{2}\right) \quad\left(\right.$ Lemma 2.3) and $p$ and $f_{2}$ are disjoint. Thus $h p f_{2}^{n+m} \in\left\langle f: \mathscr{G}_{X}\right\rangle,\left.h p\right|_{s\left(f_{2}\right)}=\left.i\right|_{S\left(f_{2}\right)}$, so $\left.h p\right|_{Z} \in \mathscr{H}(f)$.

If $q^{\prime}$ is a permutation of $Z$, let $q \in \mathscr{G}_{X}$ be such that $\left.q\right|_{z}=q^{\prime}$ and $\left.q\right|_{X-z}=\left.i\right|_{x-z}$. Let 
$\left.h\right|_{z} \in \mathscr{H}(f), h f_{2}^{n} \in\left\langle f: \mathscr{G}_{X}\right\rangle$. Then $q$ and $f_{2}$ are disjoint and $q h q^{-1} f_{2}^{n}=q h f_{2}^{n} q^{-1} \in\left\langle f: \mathscr{G}_{X}\right\rangle$, so $\left.q h q^{-1}\right|_{z} \in \mathscr{H}(f)$.

Lemma 2.5. Let $f \in W_{X}$ with shift $f=\alpha$ and $\operatorname{def} f=\beta \geqq \aleph_{0}$. Then there exists $g \in\left\langle f: \mathscr{G}_{X}\right\rangle$ such that $\mathscr{H}(g)=S\left(Y, \alpha^{+}, 0\right)$, where $Y \subseteq X$ with $|Y|=|X|$ and $|X-Y|=\beta$.

Proof. Let, as before, $f=f_{1} f_{2}, f_{1} \in \mathscr{G}_{X}, f_{2} \in C h_{X}$. Then shift $f_{2}=\beta \aleph_{0}=\beta$, and if $\alpha>\beta$ then shift $f_{1}=\alpha=|X|$ and we let $g=f, Y=X-S\left(f_{2}\right)$. The result follows from [4, 11.3.4]. Now assume $\alpha=\beta$, choose $h \in \mathscr{G}_{X}$ as in Corollary 2.2 and let $g=h f h^{-1} f=g_{1} g_{2}$, $Y=X-S\left(g_{2}\right)$. Since shift $g_{1}=\aleph_{0} \beta=\beta=\alpha$ the result follows again from $[4,11.3 .4]$.

Proposition 2.6. Let $f \in W_{X}$ with shift $f=\alpha$ and $\operatorname{def} f=\beta \geqq \aleph_{0}$. Then $\left\langle f: \mathscr{G}_{X}\right\rangle=$ $S\left(X, \alpha^{+}, \beta\right)$.

Proof. The inclusion $\left\langle f: \mathscr{G}_{X}\right\rangle \subseteq S\left(X, \alpha^{+}, \beta\right)$ follows from Lemma 1.6. To show the reverse inclusion observe that for every $p \in S\left(X, \alpha^{+}, \beta\right)$, there exist disjoint $s, t \in S\left(X, \alpha^{+}, \beta\right) \cup\left\{i_{X}\right\}$ such that $p=s t$ and the cardinalities of the sets of elements of $X$ unmoved by the chains in the cyclic-chain decompositions of $s$ and $t$ are $|X|$. Indeed, let $p=p_{1} p_{2}$, where $p_{1} \in \mathscr{G}_{X}, p_{2} \in C h_{X}, p_{1}$ and $p_{2}$ be disjoint. If $\left|X-S\left(p_{2}\right)\right|=|X|$ we let $s=p$, $t=i_{X}$. If $\left|X-S\left(p_{2}\right)\right|<|X|$ then $\left|S\left(p_{2}\right)\right|=|X|$, so that $\beta=|X|=\alpha$. Let $p_{2}=\Pi\{q: q \in A\}$, then $|A|=\beta=|X|$. Partition $A$ into sets $B$ and $C$ with $|B|=|C|=|X|$ and let $s=\Pi\{q: q \in B \cup$ $\left.\left\{p_{1}\right\}\right\}, t=\Pi\{q: q \in C\}$. Hence, to show that $\left\langle f: \mathscr{G}_{X}\right\rangle \supseteq S\left(X, \alpha^{+}, \beta\right)$ it is sufficient to show that $\left\langle f: \mathscr{G}_{X}\right\rangle$ contains all $g \in S\left(X, \alpha^{+}, \beta\right)$ with $g=g_{1} g_{2}, g_{1} \in \mathscr{G}_{X}, g_{2} \in C h_{X}, g_{1}$ and $g_{2}$ are disjoint and $\left|X-S\left(g_{2}\right)\right|=|X|$. But this follows from Lemma 2.5 and Proposition 1.5.

For a semigroup $S$ of transformations, let $\sigma$-def $S=\{\operatorname{def} f: f \in S\}$ and $\sigma$-shift $S=$ \{shift $f: f \in S\}$ be the spectrum of the defects and shifts of transformations in $S$ respectively. We are now in a position to present the first of the two theorems describing $\mathscr{G}_{X}$-normal semigroups of one-to-one transformations.

Theorem 2.7. A $\mathscr{G}_{X}$-normal semigroup $S$ of one-to-one transformations with infinite defects is a semilattice of semigroups of type $S(X, \alpha, \beta)$.

Proof. Observe that

$$
\begin{aligned}
S & =\bigcup\left\{\left\langle f: \mathscr{G}_{X}\right\rangle: f \in S\right\} \\
& =\bigcup\left\{S\left(X, \gamma^{+}, \beta\right): \gamma \in \sigma \text {-shift } S, \beta \in \sigma-\operatorname{def} S\right\},
\end{aligned}
$$

by Proposition 2.6. Also if $S\left(X, \delta_{1}, \beta_{1}\right), S\left(X, \delta_{2}, \beta_{2}\right) \subseteq S$ then

$$
\left(S\left(X, \delta_{1}, \beta_{1}\right)\right)\left(S\left(X, \delta_{2}, \beta_{2}\right)\right)=S\left(X, \delta_{1}+\delta_{2}, \beta_{1}+\beta_{2}\right)
$$

For every $\beta \in \sigma$-def $S$ let $S(\beta)=\{f \in S$ : $\operatorname{def} f=\beta\}$. Lemma 1.6 implies that $S(\beta)$ is a $\mathscr{G}_{X}$-normal subsemigroup of $S$ and so 


$$
S(\beta)=\left\{S\left(X, \gamma^{+}, \beta\right): \gamma \in \sigma \text {-shift } S(\beta)\right\}=S\left(X, \alpha_{\beta}, \beta\right),
$$

where $\alpha_{\beta}=\min \{\delta: \delta>\gamma$ for every $\gamma \in \sigma$-shift $S(\beta)\}$. Then $S=\bigcup\{S(X, \alpha, \beta): \beta \in \sigma$-def $S$, $\left.\alpha=\alpha_{\beta}\right\}$.

Now we restrict our attention to semigroups of transformations with finite defects. The structure of such semigroups is more complicated because of the variety of transformations with distinct defects presented even in a semigroup of type $\left\langle f: \mathscr{G}_{X}\right\rangle$ (Lemma 2.8). Hence, it is possible now that for given $\alpha \geqq \aleph_{0}, 0<n<\aleph_{0}$, a $\mathscr{G}_{X}$-normal $S$ contains only a proper subset of $S(X, \alpha, n)$ that does not coincide with any $S(X, \beta, n)$ for $\beta<\alpha$. We start by describing $\left\langle f: \mathscr{G}_{X}\right\rangle$ for $f$ with $\operatorname{def} f\left\langle\aleph_{0}\right.$ (Proposition 2.13). The following lemma follows from 1.6.

Lemma 2.8. Let def $f=n, 0<n<\aleph_{0}$. Then $\sigma-\operatorname{def}\left\langle f: \mathscr{G}_{X}\right\rangle=n \mathbb{N}(=\{n, 2 n, 3 n \ldots\})$.

Recall that $\phi_{k}(f)$ is the number of disjoint cycles of length $k, k \ldots=1,2, \ldots ; \infty$, in the cyclic decomposition of $f$.

Lemma 2.9. Let $h \in \mathscr{G}_{X}$ such that $\phi_{k}(h)=\gamma \geqq \aleph_{0}$ for some $k=2,3, \ldots ; \infty$. Let $U \subseteq X$ be the set that is moved by the k-cycles of $h$. Then there exists a conjugate $p$ of $h$ such that $\phi_{1}\left(\left.p h\right|_{U}\right)=\gamma, \phi_{2}\left(\left.p h\right|_{U}\right)=\gamma$ and $\phi_{l}\left(\left.p h\right|_{U}\right)=0$ for every $l=3,4, \ldots ; \infty$.

Proof. Choose a conjugate $p$ of $h$ as follows. If $k=2$, for every triple of 2-cycles of $h$ $\left(a_{1} a_{2}\right),\left(a_{3} a_{4}\right),\left(a_{5} a_{6}\right)$ let $p$ contain $\left(a_{1} a_{3}\right),\left(a_{2} a_{4}\right),\left(a_{5} a_{6}\right)$, then ph contains $\left(a_{1} a_{4}\right),\left(a_{2} a_{3}\right)$, $\left(a_{5}\right),\left(a_{6}\right)$. If $k=3$, for every pair of 3-cycles $\left(a_{1} a_{2} a_{3}\right),\left(a_{4} a_{5} a_{6}\right)$ of $h$ let $p$ contain $\left(a_{1} a_{3} a_{4}\right)$, $\left(a_{2} a_{6} a_{5}\right)$, then $p h$ contains $\left(a_{1} a_{6}\right),\left(a_{2} a_{4}\right),\left(a_{3}\right),\left(a_{5}\right)$. If $3<k<\infty$, for each $k$-cycle $\left(a_{1} a_{2} \ldots a_{k}\right)$ in $h$ let $p$ contain the $k$-cycle $\left(a_{k-3} \ldots a_{2} a_{1} a_{k-2} a_{k-1} a_{k}\right)$, then ph contains $\left(a_{1}\right)$, $\left(a_{2}\right), \ldots,\left(a_{k-4}\right),\left(a_{k-3} a_{k-1}\right),\left(a_{k-2} a_{k}\right)$. If $k=\infty$, for each infinite cycle in $h$

$$
\left(\ldots a_{-4} a_{-3} a_{-2} a_{-1} a_{0} a_{1} a_{2} a_{3} a_{4} \ldots\right)
$$

let $p$ contain the infinite cycle

$$
\left(\ldots a_{8} a_{7} a_{6} a_{3} a_{4} a_{5} a_{2} a_{1} a_{0} a_{-3} a_{-2} a_{-1} a_{-4} a_{-5} a_{-6} \ldots\right)
$$

then ph contains the following 1- and 2-cycles:

$$
\ldots\left(a_{-4} a_{-2}\right),\left(a_{-3} a_{-1}\right),\left(a_{0}\right),\left(a_{1}\right),\left(a_{2} a_{4}\right),\left(a_{3} a_{5}\right),\left(a_{6}\right),\left(a_{7}\right), \ldots
$$

The next proposition considerably eases our task of describing $\left\langle f: \mathscr{G}_{X}\right\rangle$. Namely, it shows that if there exists an integer $k$ such that $\left.\left\langle f: \mathscr{G}_{X}\right\rangle \supseteq S(X \text {, (shift } f)^{+}, k(\operatorname{def} f)\right)$ then $\left\langle f: \mathscr{G}_{X}\right\rangle \supseteq S\left(X,(\text { shift } f)^{+}, l(\operatorname{def} f)\right)$, for every $l>k$, and this describes all elements of $\left\langle f: G_{X}\right\rangle$ with defects at leas: $k(\operatorname{def} f)$. 
Proposition 2.10. Let $S$ be a $\mathscr{G}_{X}$-normal semigroup of one-to-one transformations. If there exists $n \in \sigma$-def $S$ such that $S \supseteq S(X, \alpha, n)$, for some $\alpha>\aleph_{0}$, then for every $k \in \sigma$-def $S$ with $S \cap S(X, \alpha, k-n) \neq \Phi$ we have that $S \supseteq S(X, \alpha, k)$.

Proof. Let $k$ be as stated, $f \in S(X, \alpha, k)$ and choose $t \in S \cap S(X, \alpha, k-n)$. To show that $f \in S$, it is sufficient to show that $f=s t$ for some one-to-one $s$ with shift $s<\alpha$. To construct the desired $s$ choose a one-to-one transformation $p: X-R(t) \rightarrow X-R(f)$ and for $x \in X$ let

$$
s(x)= \begin{cases}f\left(t^{-1}(x)\right) & \text { if } x \in R(t) \\ p(x) & \text { if } x \notin R(t) .\end{cases}
$$

Then $s t=f$ and we only have to show that shift $s<\alpha$. Let $D=\{x \in X: f(x) \neq t(x)\}$. Then the set of points moved by $s, S(s) \subseteq t(D) \cup(X-R(t))$ and $|D| \leqq \max$ shift $f$, shift $t\}<\alpha$. Thus $|S(s)|<\alpha$, or shift $s<\alpha$, as required.

Every permutation can be written as a product of two permutations each of which is a (formal) product of 2-cycles [4, 11.3.2]. We need a modification of this result, the proof of which is straightforward.

Lemma 2.11. Let $Z$ be an infinite set and $H$ be a normal subgroup of $\mathscr{G}_{Z}$ that contains permutations with infinite shift. Then $\forall h \in H$ there exist $p, q \in H$ with $p^{2}=i=q^{2}, h=p q$, shift $p$, shift $q \geqq \aleph_{0}$ and $S(p), S(q) \subseteq S(h)$ if shift $h \geqq \aleph_{0},|Z-S(p)|=|Z-S(q)|=|Z|$, if shift $h<\aleph_{0}$.

The next proposition together with Lemmas 2.1 and 2.11 will enable us to show that given a one-to-one $f$ with a finite defect $n$, there exists $k$ such that $\left\langle f: \mathscr{G}_{x}\right\rangle \supseteq$ $S\left(X,(\text { shift } f)^{+}, k n\right)$, and then Proposition 2.10 is applicable.

Proposition 2.12. Let $f=f_{1} f_{2}$, where $f_{1} \in \mathscr{G}_{X}, f_{2} \in C h_{X}, f_{1}$ and $f_{2}$ be disjoint and shift $f_{1}=\beta \geqq \aleph_{0}$. Let $\operatorname{def} f=n$. Then $\left\langle f: \mathscr{G}_{X}\right\rangle$ contains all one-to-one transformations $q$ such that def $q=2 n, \phi_{1}(q)=|X|, \phi_{m}(q)=0$ for $m>2$ and $\phi_{2}(q)=\gamma$, where $\aleph_{0} \leqq \gamma \leqq \beta$.

Proof. Let $q$ be as stated, $q=q_{1} q_{2}, q_{1} \in \mathscr{G}_{X}, q_{2} \in C h_{X}$. Observe that $q$ is in $\left\langle f: \mathscr{G}_{X}\right\rangle$ if and only if every conjugate of $q$ is in $\left\langle f: \mathscr{G}_{X}\right\rangle$. Hence we can assume without loss of generality that $q_{2}=f_{2}^{2}$ (else replace $q$ with its suitable conjugate). Therefore to show that $q \in\left\langle f: \mathscr{G}_{X}\right\rangle$ it is sufficient to show that there exists a conjugate $t$ of $q_{1}$ such that $t f_{2}^{2} \in\left\langle f: \mathscr{G}_{X}\right\rangle$, where $t$ and $f_{2}$ are disjoint (indeed, if $t=h q_{1} h^{-1}$ for some $h \in \mathscr{G}_{X}$ then since $q_{1}$ and $f_{2}^{2}, t$ and $f_{2}^{2}$ are disjoint, $h$ can be chosen such that $\left.h\right|_{{\mathrm{S}\left(\mathrm{S}_{2}\right)}}=\left.i\right|_{{\mathrm{S}\left(\mathrm{S}_{2}\right)}}$ and so $t f_{2}^{2}=h q_{1} h^{-1} f_{2}^{2}=h q_{1} f_{2}^{2} h^{-1}=h q h^{-1}$, a conjugate of $q$ ). To construct the required $t$, we consider two cases.

(a) $\phi_{k}\left(f_{1}\right)=\beta$ for some $1<k \leqq \infty$.

Choose a conjugate $g$ of $f_{1}$ as follows. Partition the set of the $k$-cycles of $f_{1}$ into sets 
$A$ and $B$ with $|A|=\gamma,|B|=\beta$. For the cycles in $A$, choose $g$ such that $g f_{1}$ is a product of $\gamma$ 2-cycles and 1-cycles on the points of $X$ moved by the cycles in $A$ (as in Lemma 2.9). For all the cycles of $f_{1}$ that are not in $A$, let $g$ contain their inverses. Let $t=g f_{1}$, then $t f_{2}^{2}=g f_{1} f_{2}^{2}=\left(g f_{2}\right)\left(f_{1} f_{2}\right) \in\left\langle f: \mathscr{G}_{X}\right\rangle$, and so $t$ is the required conjugate of $q_{1}$.

(b) $\phi_{k}\left(f_{1}\right)<\beta$ for every $1<k \leqq \infty$.

Then either $\beta$ is singular or the shift of $f_{1}$ is achieved by less than $\beta$ infinite cycles, so that $\beta=\gamma=\aleph_{0}$. In the latter case, take a conjugate $g$ of $f_{1}$ as follows. Choose one infinite cycle (1) in $f_{1}$ and let $g$ contain (2), then $g f_{1}$ contains (3) (as in the proof of Lemma 2.9), for the rest of the cycles of $f_{1}$ let $g$ contain their inverses. Then $t=g f_{1}$ is the required mapping.

Now assume that $\beta$ is singular. To construct a conjugate $t$ of $q_{1}$ such that $t f_{2}^{2} \in\left\langle f: \mathscr{G}_{X}\right\rangle$ we firstly assume that $\phi_{2}\left(q_{1}\right)=\gamma<\beta$. Then there exists a cardinal $\delta$ with $\gamma \leqq \delta<\beta$ such that $\phi_{k}\left(f_{1}\right)=\delta$ for some $1<k \leqq \infty$ (else $\phi_{k}\left(f_{1}\right)<\gamma$ for every $k>1$, so that $\beta=\sum_{1<k<\infty} \phi_{k}\left(f_{1}\right)+\aleph_{0} \phi_{\infty}\left(f_{1}\right) \leqq \gamma^{+}<\beta$ since $\beta$ is not a successer cardinal). Hence, to construct the requires $t$, we proceed as in (a), replacing $\beta$ by $\delta$. Now assume that $\phi_{2}\left(q_{1}\right)=\beta$. We choose a conjugate $g$ of $f_{1}$ with $g f_{1}=t$ as follows. For every $k>1$ having $\phi_{k}\left(f_{1}\right) \geqq \aleph_{0}$ partition the set of the $k$-cycles of $f_{1}$ into sets $A_{k}$ and $B_{k}$ with $\left|A_{k}\right|=\left|B_{k}\right|$. For every such $A_{k}$, let $g$ be such that $g f_{1}$ is a product of $\phi_{k}\left(f_{1}\right) 2$-cycles and 1-cycles on the points of $X$ moved by the cycles in $A_{k}$ (as in Lemma 2.9). For all the cycles of $f_{1}$ that are not in $\left\{A_{k}: \phi_{k}\left(f_{1}\right) \geqq \aleph_{0}\right\}$ let $g$ contain their inverses.

The proposition below describes elements of a semigroup $S=\left\langle f: \mathscr{G}_{X}\right\rangle$, where $f$ is a one-to-one transformation with a finite defect $n$. Informally, the statement of the proposition means that there exists an integer $k_{0} \in \sigma$-def $S$ such that for every $k \in \sigma-\operatorname{def} S$ with $k \geqq k_{0}$, the set of transformations in $S$ with defect $k$ consists of all $1-1$ mappings with defect $k$ that shift at most as many points of $X$ as $f$ does.

Proposition 2.13. Let $f \in S\left(X,|X|^{+}, n\right), f=f_{1} f_{2}$ with $f_{1} \in \mathscr{G}_{X}, f_{2} \in C h_{X}, f_{1}$ and $f_{2}$ be disjoint. If $\aleph_{0} \leqq$ shift $f_{1}<|X|$, then for every integer $k \geqq 4$,

$$
\left\langle f: \mathscr{G}_{X}\right\rangle \cap S\left(X,|X|^{+}, k n\right)=S\left(X,\left(\text { shift } f_{1}\right)^{+}, k n\right) .
$$

If shift $f_{1}=|X|$, then if $|X|>\aleph_{0}$ and $k \geqq 4$, we have

$$
\left\langle f: \mathscr{G}_{X}\right\rangle \supseteq S(X,|X|, k n),
$$

and if $|X| \geqq \aleph_{0}$ and $k \geqq 8$ then

$$
\left\langle f: \mathscr{G}_{X}\right\rangle \supseteq S\left(X,|X|^{+}, k n\right) .
$$

If shift $f_{1}<\aleph_{0}$ and $|X|>\aleph_{0}$, then for every integer $k \geqq 4$,

$$
\left\langle f: \mathscr{G}_{X}\right\rangle \cap S\left(X,|X|^{+}, k n\right)=S\left(X, \aleph_{0}, k n\right),
$$


and if shift $f_{1}<\aleph_{0}$ and $|X|=\aleph_{0}$, then equality (7) holds for every integer $k \geqq 8$.

Proof. Take $g \in S\left(X,|X|^{+}, k n\right)$ and write $g=g_{1} g_{2}$ with $g_{1} \in \mathscr{G}_{X}, g_{2} \in C h_{X}$ and $g_{1}, g_{2}$ are being disjoint. Our aim is to show that $g \in\left\langle f: \mathscr{G}_{X}\right\rangle$ whenever $k$ is sufficiently large (as described in the statement of the theorem) and shift $g$ does not exceed shift $f$. Also Proposition 2.10 allows to reduce the proofs to the case $k=4[k=8]$ whenever the statement of the theorem involves $k \geqq 4[k \geqq 8]$. We consider the following cases.

Case 1: shift $f_{1} \geqq \aleph_{0}$.

Suppose firstly that $\left|X-S\left(g_{2}\right)\right|=|X|\left(=\left|X-S\left(f_{2}\right)\right|\right)$ (if $|X|>\aleph_{0}$ this is always true). Then in view of Proposition 1.5, we can assume that $g_{2}=f_{2}^{k}$. We set $X-S\left(f_{2}\right)=Z$ and prove firstly the following.

Claim. If $\left|Z-S\left(g_{1}\right)\right|=|X|$ and $\operatorname{def} g=4 n$ then $g \in\left\langle f: \mathscr{G}_{X}\right\rangle$.

Indeed, using Lemma 2.11 we can write $g_{1}=p q$, where $p, q \in \mathscr{G}_{X}, p^{2}=i=q^{2}$, shift $p$, shift $q \geqq \boldsymbol{N}_{0}, S(p), S(q) \subseteq Z$ and $|Z-S(p)|=|Z-S(q)|=|X|$. Then $g=g_{1} g_{2}=p q f_{2}^{4}=\left(p f_{2}^{2}\right)$ $\left(q f_{2}^{2}\right)$ and Proposition 2.12 ensures that $p f_{2}^{2}, q f_{2}^{2} \in\left\langle f: \mathscr{G}_{X}\right\rangle$, so that $g \in\left\langle f: \mathscr{G}_{X}\right\rangle$.

Now (4) and (5) follow from the above claim and the observation that shift $g_{1}<|X|$ together with $\left|X-S\left(g_{2}\right)\right|=|X|$ imply that $\left|Z-S\left(g_{1}\right)\right|=|X|$. To prove (6), observe that using Lemma 2.11 repeatedly, we can find $t_{1}, t_{2}, t_{3}, t_{4} \in \mathscr{G}_{X}$ such that for $j=1,2,3,4$, $t_{j}^{2}=i$, shift $t_{j} \geqq \aleph_{0}, S\left(t_{j}\right) \subseteq Z,\left|Z-S\left(t_{j}\right)\right|=|X|$ and $g_{1}=t_{1} t_{2} t_{3} t_{4}$, so that $g=g_{1} g_{2}=$ $t_{1} t_{2} t_{3} t_{4} f_{2}^{8}=\left(t_{1} f_{2}^{2}\right)\left(t_{2} f_{2}^{2}\right)\left(t_{3} f_{2}^{2}\right)\left(t_{4} f_{2}^{2}\right) \in\left\langle f: \mathscr{G}_{X}\right\rangle$.

Now assume that $\left|X-S\left(g_{2}\right)\right|<\aleph_{0}$, then $|X|=\aleph_{0}$ and we only have to show the statement (6). Write $g_{2}=t s$ where $t$ and $s$ are disjoint chains with def $t=\operatorname{def} s=4 n$. It follows easily from the claim above that both $g_{1} t$ and $s$ are in $\left\langle f: \mathscr{G}_{X}\right\rangle$ and so that $g=g_{1} g_{2}=\left(g_{1} t\right) s \in\left\langle f: \mathscr{G}_{X}\right\rangle$.

Case 2. shift $f_{1}<\boldsymbol{N}_{0}$.

Since shift $f_{2}=\aleph_{0}$, the above assumption implies that shift $f=\aleph_{0}$. We start by showing that $\left\langle f: \mathscr{G}_{X}\right\rangle$ contains all transformations that are (formal) products of $2 n$ disjoint chains and countably many disjoint two-cycles and that fix $|X|$ points of $X$. For that choose a conjugate $q$ of $f$ such that for every cycle of $f$ the transformation $q$ contains its inverse while for every chain $f^{\prime}$ of $f, q$ contains a chain $q^{\prime}$ with $S\left(f^{\prime}\right)=S\left(q^{\prime}\right)$ and $q^{\prime} f^{\prime}$ is being a product of two disjoint chains, infinitely many disjoint 2-cycles and fixing $\aleph_{0}$ points of $S\left(f^{\prime}\right)$ (Lemma 2.1). Thus, $q f \in\left\langle f: \mathscr{G}_{X}\right\rangle$ with def $q f=2 n, \phi_{2}(q f)=\aleph_{0}$, $\phi_{1}(q f)=|X|$ and $\phi_{m}(q f)=0$ for $m \geqq 3$. Then the rest of the proof is analogous to that of Case 1 .

The following summarizes the results of 2.13 .

Corollary 2.14. Let $f \in S\left(X,|X|^{+}, n\right), f=f_{1} f_{2}$ with $f_{1} \in \mathscr{G}_{X}, f_{2} \in C h_{X}, f_{1}$ and $f_{2}$ be disjoint. Let shift $f_{1}=\beta, A$ be the set of all conjugates of $f, \alpha=\max \left\{\beta^{+}, \aleph_{1}\right\}$. Then there exists an integer $k$ that is determined by $f, k=k(f)$, such that 


$$
\left\langle f: \mathscr{G}_{X}\right\rangle=\bigcup_{i=1}^{k(f)} A^{i} \bigcup\{S(X, \alpha, m n): m>k(f)\},
$$

with $k(f) \leqq 3$ if either $\aleph_{0} \leqq \beta<|X|$ or $\beta<\aleph_{0}$ and $|X| \geqq \aleph_{0}$, and $k(f) \leqq 7$ otherwise.

If $S$ is a $\mathscr{G}_{X}$-normal semigroup of one-to-one transformations with finite defects then $\sigma$-def $S$ forms a subsemigroup of the semigroup $(\mathbb{N},+)$ of all natural numbers with respect to addition. A semigroup $T \subseteq(\mathbb{N},+)$ can be partially ordered such that for $m$, $n \in T, m \leqq n(T)$ if $n-m \in T \cup\{0\}$. The set of minimal non-zero elements of $T$ generates $T$ and is called a minimal set of generators for $\mathscr{T}$. The set of minimal generators is finite and its cardinality does not exceed the smallest non-zero $n \in T$ [1]. Hence, the following proposition.

Proposition 2.15. $\sigma$-def $S=\left\langle n_{1}, \ldots, n_{l}\right\rangle$ with $l<\min \left\{n_{i}\right\}, i=1, \ldots, l$.

Our aim now is to describe elements of $S$. Let

$$
\mu=\min \{\beta: \beta>\text { shift } f \text { for every } f \in S\} .
$$

Then $\mu \leqq|X|^{+}$and $\sigma$-shift $S=\left[\aleph_{0}, \mu\right)$. Also $S \subseteq \bigcup\{S(X, \mu, n): n \in \sigma$-def $S\}$. The reverse inclusion is not true in general. Below we determine for which $n \in \sigma$-def $S, \alpha \in\left(\aleph_{0}, \mu\right]$, $S \supseteq S(X, \alpha, n)$. For every infinite cardinal $\alpha<\mu$ let

$$
\tau(\alpha)=\min \{\text { def } f: f \in S \text { with shift } f=\alpha\} .
$$

The following result follows from Propositions 2.13 and 2.10 .

Proposition 2.16. $S \supseteq S\left(X, \alpha^{+}, n\right)$ for every $n \in \sigma-\operatorname{def} S$ such that $n \geqq 8 \tau(\alpha)(\sigma-\operatorname{def} S)$.

For every $\alpha \in\left[\aleph_{0}, \mu\right]$ let

$$
\mathscr{A}_{\alpha}=\left\{f \in S: \text { shift } f<\alpha^{+}\right\}
$$

Clearly $\mathscr{A}_{\alpha} \subseteq \mathscr{A}_{\beta}$ iff $\alpha \leqq \beta$. Our next result describes $S$ in terms of its subsemigroups $\mathscr{A}_{\alpha}$. It is convenient to extend the definition of a $\mathscr{G}_{X}$-normal semigroup to a subset $A$ of transformations of $X$ saying that $A$ is normal if $h A h^{-1}=A$ for every $h \in \mathscr{G}_{X}$.

Theorem 2.17. $A \mathscr{G}_{X}$-normal semigroup $S$ of one-to-one transformations with finite defects is a chain of its subsemigroups $\mathscr{A}_{\alpha}, \alpha \in\left[\aleph_{0}, \mu\right)$. For every $\alpha$ there exist an integer $k=k(\alpha)$, a finite index set $I_{\alpha}=\left\{i_{1}, i_{2}, \ldots, i_{m}\right\} \subseteq \sigma$-def $S$ (with $i_{j}<i_{j+1}, j=1, \ldots, m-1$ ) and normal sets $A_{i_{j}} \subseteq S\left(X, \alpha^{+}, i_{j}\right), i_{j} \in I_{\alpha}$ with $A_{i_{j}}^{2} \subseteq A_{i_{j+1}}$ such that

$$
\mathscr{A}_{\alpha}=\bigcup\left\{A_{i}, i_{j} \in I_{\alpha}\right\} \bigcup\left\{S\left(X, \alpha^{+}, d m\right): m \geqq k(\alpha) d^{-1}\right\},
$$


where $d=\left(n_{1}, n_{2}, \ldots, n_{l}\right)$ and $\left\{n_{1}, n_{2}, \ldots, n_{l}\right\}$ is the minimal set of generators for $\sigma$-def $S$.

Proof. Let $\left\{n_{1}, n_{2}, \ldots, n_{l}\right\}$ and $d$ be as above. The semigroup $\left\langle n_{1} d^{-1}, n_{2} d^{-1}, \ldots, n_{1} d^{-1}\right\rangle$ is isomorphic to $\sigma$-def $S$ and has a finite complement in $\mathbb{N}$ [1]. The Frobenius number $F$ of $\left\langle n_{1} d^{-1}, n_{2} d^{-1}, \ldots, n_{l} d^{-1}\right\rangle$ is the maximal integer that is not in the semigroup. Thus for every $s>F$, sd $\in \sigma$-def $S$. Let $k(\alpha)=\min (\{s d: s>F\} \cap\{t \tau(\alpha): t>7\})$. Proposition 2.13 ensures that

$$
\mathscr{A}_{\alpha} \supseteq\left\{S\left(X, \alpha^{+}, d m\right): m \geqq k(\alpha) d^{-1}\right\} .
$$

Let $I_{\alpha}=\{i \in \sigma$-def $S: i<k(\alpha)\}$, and for each $i \in I_{\alpha}$, let $A_{i}=S \cap S\left(X, \alpha^{+}, i\right)$. The result follows by applying Proposition 2.10 .

\section{REFERENCES}

1. R. Fröberg, C. Gottlieb and R. Häggkvist, On numerical semigroups, Semigroup Forum 35 (1987), 63-83.

2. I. Levi, Automorphisms of normal transformation semigroups, Proc. Edinburgh Math Soc. 28 (1985), 185-205.

3. B. M. Schein, Symmetric semigroups of transformations, Abstracts Amer. Math. Soc. 5 (1980), 476.

4. W. R. Scotr, Group Theory (Prentice-Hall, 1964).

Department of Mathematics

UNIVERSITY OF LOUISVILLE

LOUISVILLE, KY 40292

U.S.A. 